\section{DEVELOPMENT}

\section{Dendrites need guidance too}
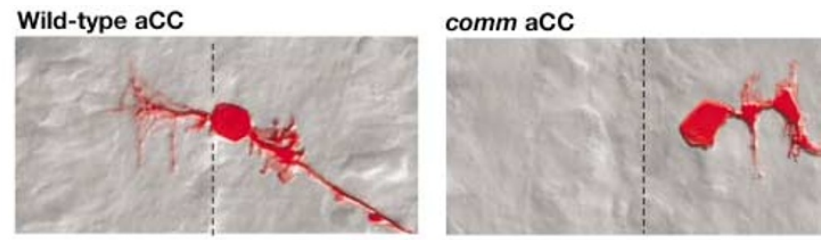

\section{Wild-type RP3}

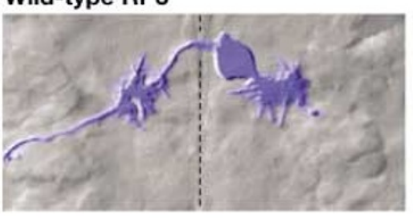

robo RP3

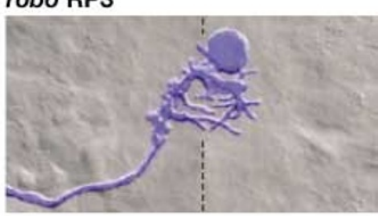

In wild-type Drosophila embryos, the aCC (red) and RP3 (blue) neurons extend dendrites on both sides of the midline (dashed line). In the comm mutant, the aCC dendrites that normally cross the midline are lost or reduced. In the robo mutant, the RP3 dendrites grow towards the midline and often wrap around it. Reproduced, with permission, from Furrer et al., Nature Neuroscience @ (2003) Macmillan Magazines Ltd.

The importance of precise axon guidance for the wiring of the nervous system has long been recognized, and studies in flies and vertebrates have brought to light a multitude of molecules that allow growth cones to navigate the developing nervous system. But axons are not the only neuronal projections that need guidance, as Furrer et al. report in Nature Neuroscience. Dendrites also need to be accurately steered to their targets to make appropriate synaptic connections, and this study shows that they rely on some of the same cues as axons.

In the Drosophila central nervous system, two sets of molecules help to determine whether axons project contralaterally (across the midline) or ipsilaterally (away from or parallel to the midline). Slit and its receptor Robo mediate repulsion at the midline, thereby preventing axons from crossing, whereas Netrin and its receptor Frazzled mediate attraction, allowing axons to cross the midline to form commissural tracts. Furrer et al. asked whether these molecules also regulate midline crossing of dendrites. In addition, they studied the effects on dendritic guidance of mutations in commissureless (comm), a gene that codes for a protein that counteracts Slit/Robo-mediated repulsion.
In comm mutants, the number of contralaterally-projecting axons is considerably reduced. Furrer et al. now show that many populations of dendrites that would normally project contralaterally also fail to cross the midline in these mutants. A similar phenomenon was seen in netrin and frazzled mutant embryos. In robo mutants, the commissures are thickened, and it was previously thought that this was largely due to increased crossing and re-crossing of axons. However, Furrer et al. show that aberrant crossing of dendrites also contributes significantly to this phenotype.

The dendritic guidance defects in frazzled and robo mutants could be rescued in individual neurons by restoring the function of the corresponding gene, indicating that Frazzled and Robo act cellautonomously to regulate the response of growing dendrites to guidance cues in their environment. The authors also found that in rescued frazzled mutants, Frazzled protein was found in dendrites that

SYSTEMS NEUROSCIENCE

\title{
Ghrelin on the brain
}

The elusive source of endogenous ghrelin in the hypothalamus is described by Horvath, Cowley and colleagues in a report in Neuron. Ghrelin is a peptide hormone that is produced mainly by the stomach in response to fasting. It activates the growth hormone secretagogue receptor and has powerful actions on feeding and energy regulatory circuits, including a direct influence on hypothalamic neurons. The new results indicate that at least some of these effects might be mediated by brain-derived ghrelin, rather than by ghrelin from the stomach.

Cowley et al. showed that ghrelin is expressed in a previously uncharacterized group of neurons in the hypothalamus. The neurons lie in the space between the lateral hypothalamic, arcuate, ventromedial, dorsomedial and paraventricular hypothalamic nuclei, and they send projections to several of these nuclei as well as outside the hypothalamus. Interestingly, the area that contains the ghrelin neurons overlaps with the projections from the suprachiasmatic nucleus, which might allow the production of ghrelin to be directly modulated by the circadian clock.

Although there have been previous indications that ghrelin is produced in the hypothalamus, the function of this brainderived ghrelin is unclear. Cowley and colleagues have provided some clues to what it might be doing. Anatomically, they saw that ghrelin-containing boutons are often closely apposed to the axon terminals of other hypothalamic neurons, particularly those containing neuropeptide Y (NPY), raising the possibility that it might act presynaptically to modulate the release of NPY and GABA ( $\gamma$-aminobutyric acid) by these neurons.

The authors went on to investigate the physiological effects of ghrelin in two hypothalamic nuclei, the arcuate nucleus and the paraventricular nucleus. They found that treatment with ghrelin increases the activity of the NPY-containing neurons to which the ghrelin neurons project, and hyperpolarizes pro-opiomelanocortin (POMC)-containing neurons in the arcuate nucleus. This hyperpolarization is probably mediated by increased release of NPY and GABA.

In the paraventricular nucleus, ghrelin reduced the GABA-mediated inhibition of $73 \%$ of neurons, but did not affect the rest. Previous work showed that NPY had the same effect, and the authors propose that the influence of ghrelin here is also mediated by a presynaptic increase in NPY release, which might in turn act presynaptically to reduce GABA secretion.

The existence of an endogenous ghrelin system in the hypothalamus might solve some of the problems that have been associated with the idea that ghrelin from the stomach acts on these nuclei to regulate energy homeostasis. If more work confirms the functions of the ghrelin-containing hypothalamic neurons, it will represent an important step in our understanding of how complex interactions between the brain and the gastrointestinal system regulate our food intake and energy expenditure.

Rachel Jones

(2) References and links ORIGINAL RESEARCH PAPER Cowley, M. A. et al. The distribution and mechanism of action of ghrelin in the CNS demonstrates a novel hypothalamic circuit regulating energy homeostasis. Neuron 37, 649-661 (2003)

FURTHER READING Inui, A. Ghrelin: an orexigenic and somatotrophic signal from the stomach. Nature Rev. Neurosci. 2, 551-560 (2001) 
were able to cross the midline, whereas in rescued robo mutants, Robo was localized to dendrites that were repelled by the midline.

These findings raise an intriguing question about the regulation of Robo and Frazzled expression in developing neurons. Many neurons extend several axonal and dendritic processes, of which some project contralaterally and some ipsilaterally. Together with the observations on the subcellular localization of Robo and Frazzled in rescued mutant neurons, this indicates that Robo and Frazzled are probably targeted differentially to processes that emanate from the same cell. The question of how this is achieved will undoubtedly provide a focus for future investigations.

Heather Wood

(4) References and links ORIGINAL RESEARCH PAPER Furrer, M.-P. et al. Robo and Frazzled/DCC mediate dendritic guidance at the CNS midline. Nature Neurosci. 6 , 223-230 (2003)

FURTHER READING Yu, T. W. \& Bargmann, C. I. Dynamic regulation of axon guidance. Nature Neurosci. 4, 1169-1176 (2001)

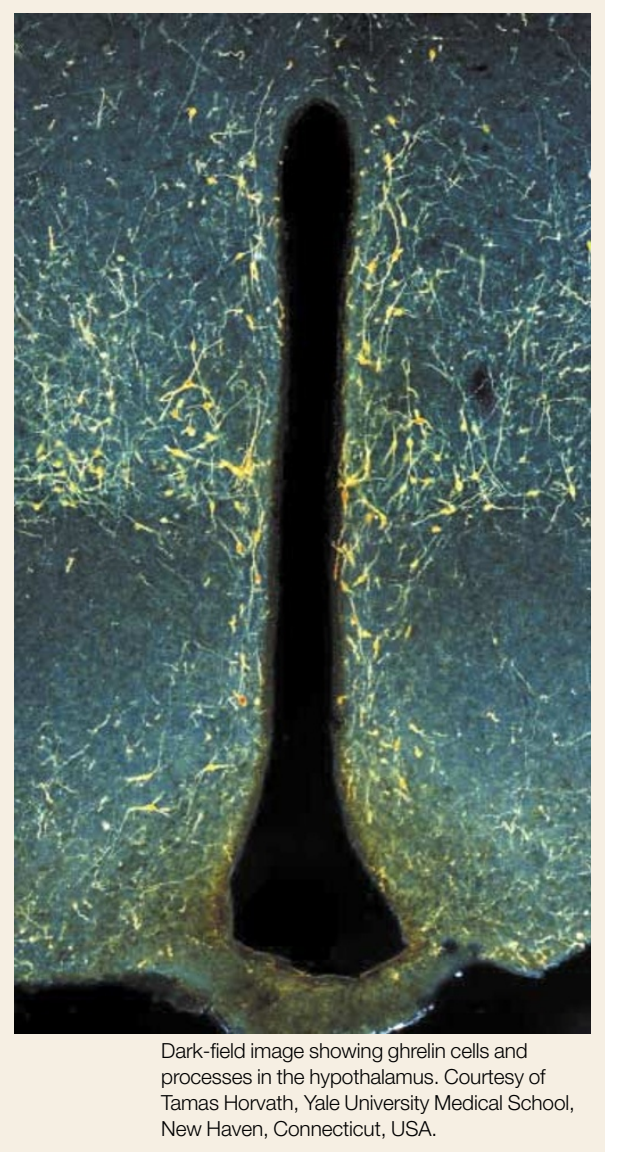

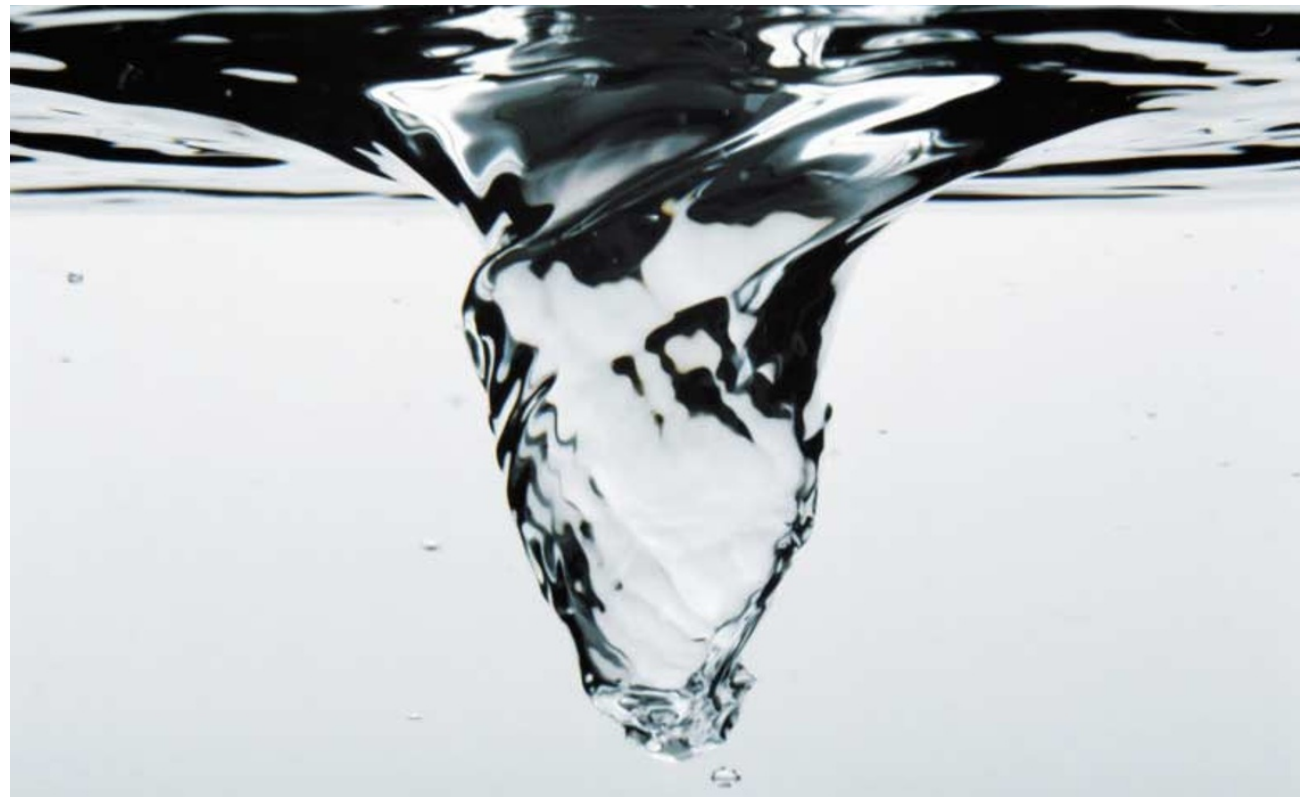

BLOOD-BRAIN BARRIER

\section{Water in the brain}

In the one-week period after a stroke, cerebral oedema - swelling of brain tissue due to the rapid influx of water - is one of the factors most likely to cause death. The development of efficient therapies for this condition has been hampered by a lack of information regarding the route of water flow into the brain. But new data from AmiryMoghaddam and colleagues implicates the water channel AQP4 in water flow across the interface between blood and brain.

AQP4 is preferentially localized to the astrocyte membrane domains that form the interface between the brain and the extracerebral liquid spaces (which comprise the cerebral microvessels and subarachnoidal space). AQP4 is anchored in these domains by an adaptor protein, $\alpha$-syntrophin - knocking out the expression of $\alpha$-syntrophin in mice decreases the density of AQP4 in these brain regions at least eight-fold. Using $\alpha$-syntrophin knockout mice, AmiryMoghaddam et al. temporarily occluded the middle cerebral artery to induce ischaemia. After reperfusion of the occluded artery, the volume of cerebral oedema was significantly reduced relative to wild type, indicating that water influx through AQP4 is a key regulator of ischaemiainduced oedema.

As AQP4 is thought to facilitate the bidirectional movement of water, the authors wondered if its capacity to facilitate water efflux from the brain influenced the rate at which swelling dissipates following ischaemic insult. This point is of particular therapeutic interest as swelling is slow to subside after stroke, significantly prolonging the risk of long-term damage. Immunolabelling of wild-type brains using anti- $\mathrm{AQP} 4$ antibodies revealed that $\mathrm{AQP} 4$ expression is virtually abolished in the core of damaged tissue after occlusion and reperfusion of the cerebral artery. AQP4 levels were also significantly reduced in the ischaemic penumbra, an area of affected tissue immediately adjacent to the anoxic core that, although threatened, can be rescued if blood flow is rapidly restored. Interestingly, $\alpha$-syntrophin levels were unchanged in these areas under the same conditions, indicating that the coupling between this adaptor molecule and AQP4 is sensitive to ischaemia and reperfusion.

It is somewhat surprising that the expression of a water channel that has the potential to hasten the reduction of swelling by facilitating the efflux of water should be decreased in response to the very cause of this damaging oedema. The authors postulate that this downregulation of AQP4 might occur during the early stages of ischaemia as a means of limiting the initial influx of water, thereby keeping swelling to a minimum. AQP4 could therefore be a useful target for the development of drug therapies that aim to minimise cerebral oedema. But will the extent by which swelling is minimised by such treatments compensate for the inevitable prolongation of the recovery period? This physiological dilemma - a consequence of the capacity of AQP4 to facilitate both the influx and efflux of water - will need to be taken into consideration if the expression of this regulatory water channel is to be therapeutically manipulated.

Suzanne Farley

(D) References and links

ORIGINAL RESEARCH PAPER Amiry-Moghaddam, M. et al. An $\alpha$-syntrophin-dependent pool of AQP4 in astroglial end-feet confers bidirectional water flow between blood and brain. Proc. Natl Acad. SCi. USA 100, 2106-2111 (2003)

Encyclopedia of Life Sciences: http://www.els.net stroke | water channels 